\title{
Polar motion of Titan forced by the atmosphere
}

\author{
Tetsuya Tokano, ${ }^{1}$ Tim Van Hoolst, ${ }^{2}$ and Özgür Karatekin ${ }^{2}$ \\ Received 12 October 2010; revised 19 January 2011; accepted 25 February 2011; published 13 May 2011.
}

[1] Titan's atmosphere possesses an equatorial component of angular momentum, which can be transferred to the surface and can excite polar motion of Titan. The atmospheric excitation of Titan's polar motion is calculated using the wind and pressure data prediction from a general circulation model. The polar motion equation is solved considering Titan's triaxial shape and different hypothetical interior models. Titan's polar motion basically consists of a superposition of small diurnal wobbles and larger semiannual and annual wobbles caused by seasonal redistribution of wind and pressure pattern. If the entire interior of Titan is solid, the polar motion has total amplitudes of a few meters, but the diurnal and seasonal wobble may be intermingled due to preferential damping of the seasonal wobble by Saturn's gravitational torque. If instead there is a subsurface ocean underneath the crust, the wobble amplitude could be larger by an order of magnitude. If the crust is thin, a resonance between the seasonal and Chandler wobble further increases the polar motion amplitude and makes the polar motion path elliptical. The seasonal wobble of a crust lying on a subsurface ocean experiences damping by either gravitational and pressure torque or elastic torque, but the relative reduction of the polar motion amplitude by these torques is likely to be smaller than that of the length-of-day variations.

Citation: Tokano, T., T. Van Hoolst, and Ö. Karatekin (2011), Polar motion of Titan forced by the atmosphere, J. Geophys. Res., 116, E05002, doi:10.1029/2010JE003758.

\section{Introduction}

[2] Saturn's largest moon Titan is covered by a dense atmosphere of $\sim 1.5$ bars. Such a dense atmosphere could appreciably change the rotation rate or length of day (LOD) of Titan due to a periodical exchange of angular momentum between the surface and atmosphere [Tokano and Neubauer, 2005; Friedson et al., 2009]. Observations of Titan's rotational state by Cassini have been used in combination with model predictions [Tokano and Neubauer, 2005] to constrain the interior structure of Titan, particularly the presence or absence of an internal ocean [Lorenz et al., 2008]. Subsequent theoretical studies [Karatekin et al., 2008; Van Hoolst et al., 2009; Mitchell, 2009; Goldreich and Mitchell, 2010] discussed how various additional torques would suppress the large LOD variation or cause a seasonal shift with respect to the prediction by Tokano and Neubauer [2005].

[3] All these studies considered only the relationship between the axial component of the atmospheric angular momentum (AAM) and Titan's rotation rate. However, the AAM is a three-dimensional vector, which can contain equatorial components in addition to the axial (polar) component [Barnes et al., 1983]. Cassini observations of Titan's atmosphere revealed a $4^{\circ}$ tilt of the symmetry axis of the stratospheric atmospheric circulation from the polar axis and

\footnotetext{
${ }^{1}$ Institut für Geophysik und Meteorologie, Universität zu Köln, Cologne, Germany.

${ }^{2}$ Royal Observatory of Belgium, Brussels, Belgium.

Copyright 2011 by the American Geophysical Union. 0148-0227/11/2010JE003758
}

its westward rotation [Achterberg et al., 2008; Roman et al., 2009; Teanby et al., 2010]. This is direct evidence of an equatorial component of the AAM on Titan. Subsequently, the presence of a nonzero equatorial AAM and its westward rotation (precession) was also recognized in the output of the Titan general circulation model (GCM) of Tokano [2010]. This was ascribed to thermal tides and the same GCM also predicted a seasonal variation in the tilt angle or, equivalently, the amount of the equatorial AAM.

[4] An important effect of the time variation of the equatorial AAM is the polar motion or wobble of the underlying body [e.g., Munk and MacDonald, 1960; Barnes et al., 1983]. The polar motion is the motion of the planetary rotation axis across its surface and has been observed on Earth [Munk and MacDonald, 1960] and Mars [Konopliv et al., 2006]. Considering the large AAM of Titan, a similar effect can also be expected on Titan as tentatively suggested by Bills and Nimmo [2008]. This effect, however, has not yet been quantified. It fundamentally differs from the secular wobble forced by the spin-orbit synchronization calculated by Noyelles et al. [2008] and Noyelles [2008].

[5] This study aims at numerically quantifying the polar motion of Titan using the output of the Titan GCM of Tokano [2010] and taking into account Titan's shape and various models of Titan's interior [e.g., Sotin et al., 2009]. This is the logical next step once the relationship between the axial AAM and LOD variation is addressed and the equatorial AAM is quantified. We expect that this calculation is not only important in its own right, but can contribute to the investigation of Titan's interior structure in combination with observational data. Our approach is analogous to 
Table 1. Parameters of Titan's Interior Calculated With the Gravity Data of Iess et al. [2010]

\begin{tabular}{lcc}
\hline \multicolumn{1}{c}{ Parameter } & Symbol & Value \\
\hline & Iess et al. [2010] & \\
Mean radius (m) & $R$ & $2.575 \times 10^{6}$ \\
Degree 2 order 0 gravity coefficient & $J_{2}$ & $3.18 \times 10^{-5}$ \\
Degree 2 order 2 gravity coefficient & $C_{22}$ & $9.98 \times 10^{-6}$ \\
Degree 2 order 2 gravity coefficient & $S_{22}$ & $0.22 \times 10^{-6}$ \\
Axial MOI factor & $C /\left(M R^{2}\right)$ & 0.34
\end{tabular}

\begin{tabular}{lcc}
\multicolumn{1}{c}{ This Study } & & \\
Axial MOI $\left(\mathrm{kg} \mathrm{m}^{2}\right.$ ) & $C$ & $3.03218 \times 10^{35}$ \\
Semimajor equatorial MOI $\left(\mathrm{kg} \mathrm{m}^{2}\right)$ & $A$ & $3.03172 \times 10^{35}$ \\
Semiminor equatorial MOI $\left(\mathrm{kg} \mathrm{m}^{2}\right)$ & $B$ & $3.03208 \times 10^{35}$ \\
Polar flattening & $\alpha$ & $9.355 \times 10^{-5}$ \\
Load Love number (no ocean) & $k_{2}^{\prime}$ & -0.03 \\
Load Love number (with ocean) & $k_{2}^{\prime}$ & -0.83 \\
Tidal potential Love number (no & $k_{2}$ & 0.03 \\
$\quad$ ocean) & & \\
Tidal potential Love number (with & $k_{2}$ & $0.28-0.4$ \\
$\quad$ ocean) & & $3.25132 \times 10^{-10}$ \\
$\begin{array}{l}\text { Chandler frequency (no ocean) }(\mathrm{Hz}) \\
\text { Chandler period (no ocean) (years) }\end{array}$ & $\sigma_{C W}$ & 612.4 \\
\hline
\end{tabular}

the calculation of Mars' polar motion by Van den Acker et al. [2002] and Sanchez et al. [2004].

[6] Section 2 describes the methods of our study, i.e., the description of the physics, of the mathematical treatment and of the model used. Section 3 presents the various results of the calculation including the atmospheric forcing, parameters of Titan's interior relevant for the polar motion and the polar motion itself. Section 4 discusses how the polar motion could be modified by additional effects.

\section{Methods}

\subsection{Chandler Wobble}

[7] In general, the polar motion of a planet/moon is a combination of the Chandler wobble and wobbles forced by geophysical fluids such as atmosphere or ocean. The Chandler wobble is the free wobble of a nonrigid planet/ moon, which owes its existence to the misalignment of the rotation axis and the figure axis. Its period/frequency is solely determined by the shape and interior structure of the body and is fully independent of the atmospheric or oceanic forcing.

[8] The angular location of the instantaneous spin pole with respect to the principal axes of Titan can be represented by the complex function $m(t)=m_{x}(t)+i m_{y}(t)$. By analogy with the geodetic definition for the Earth, we define the $\mathrm{x}$ component of $m$ as being directed toward Saturn $\left(0^{\circ}\right.$ longitude) at Titan's pericenter and the y component toward the equator at $90^{\circ} \mathrm{E}$.

[9] Following this convention, the three principal momenta of inertia (MOI) are defined as $C$ (axial), $A$ (semimajor equatorial, along the $\mathrm{x}$ axis) and $B$ (semiminor equatorial, along the y axis). The equatorial principal MOI, $A$ and $B$, are determined as

$$
\begin{aligned}
& A=C-J_{2} M R^{2}-2 M R^{2} \sqrt{C_{22}^{2}+S_{22}^{2}} \\
& B=C-J_{2} M R^{2}+2 M R^{2} \sqrt{C_{22}^{2}+S_{22}^{2}}
\end{aligned}
$$

where $M=1.345 \times 10^{23} \mathrm{~kg}$ is Titan's mass and $R=2575 \mathrm{~km}$ is Titan's mean radius. The gravity coefficients of Titan $\left(J_{2}, C_{22}\right.$ and $\left.S_{22}\right)$ have been determined by the Cassini radio science [Iess et al., 2010] and are also listed in Table 1.

[10] The equations of the Chandler wobble $m_{x}$ and $m_{y}$ of Titan considering the triaxial shape can be derived from the Liouville equations, which describe the change in angular momentum of a deformable planet/moon due to an applied torque [Van Hoolst, 2007], as

$$
\begin{aligned}
& A \Omega \dot{m}_{x}+\Omega^{2}(C-B) m_{y}+k_{2} \Omega \frac{\Omega^{2} R^{5}}{3 G} \dot{m}_{x}-k_{2} \Omega^{2} \frac{\Omega^{2} R^{5}}{3 G} m_{y}=0 \\
& B \Omega \dot{m}_{y}-\Omega^{2}(C-A) m_{x}+k_{2} \Omega \frac{\Omega^{2} R^{5}}{3 G} \dot{m}_{y}+k_{2} \Omega^{2} \frac{\Omega^{2} R^{5}}{3 G} m_{x}=0
\end{aligned}
$$

where $\Omega=4.56 \times 10^{-6} \mathrm{~s}^{-1}$ is Titan's angular velocity, $k_{2}$ is the degree 2 tidal potential Love number describing the resistance of the body to deformation (see section 3.1) and $G$ is the universal gravitational constant.

[11] The Chandler wobble frequency is the eigenfrequency of equations (3) and (4):

$$
\sigma_{C W}=\sqrt{\frac{C-\widetilde{A}}{\widetilde{B}} \frac{C-\widetilde{B}}{\widetilde{A}}} \Omega
$$

where

$$
\widetilde{A}=A+k_{2} \frac{\Omega^{2} R^{5}}{3 G}
$$

$$
\widetilde{B}=B+k_{2} \frac{\Omega^{2} R^{5}}{3 G}
$$

describe the equatorial MOI modified by a mass redistribution of deformable Titan due to the variable centrifugal potential.

[12] Equation (5) extends the classical expression for the Euler frequency for a triaxial body (for which $A<B<C$ ) by including the effect of deformation and is equivalent to the expression of Van Hoolst and Dehant [2002] up to the order in the flattenings considered by them. Equation (5) applies if Titan has no decoupling subsurface ocean.

[13] If there is a subsurface ocean and the overlying crust can be assumed to be decoupled from the deeper interior, the Chandler wobble frequency inversely scales with the effective MOI, so equation (5) has to be multiplied by $C / C_{c}$, where $C_{c}$ is the polar MOI only of the outer crust. $C_{c}$ is a function of the crustal thickness, which is a free parameter in the model, and is calculated as

$$
C_{c}=\frac{8}{15} \pi \rho_{c}\left(R^{5}-c_{c}^{5}\right)\left(1+\frac{2}{3} \alpha\right)
$$

where $\rho_{c}=917 \mathrm{~kg} \mathrm{~m}^{-3}$ is the density of the outer crust (ice I), $c_{c}$ is the inner radius of the crust (radius of the crust-ocean interface), i.e., $R-c_{c}$ is the crustal thickness, and $\alpha$ is the dynamical polar flattening. Equation (8) is modified from equation (8) of Sohl et al. [2003]. The factor $2 / 3 \alpha$ accounts for the relative difference between the mean and polar MOI. 
[14] The dynamical polar flattening is defined as

$$
\alpha=\frac{C-\frac{A+B}{2}}{\frac{A+B}{2}}
$$

\subsection{Atmospheric Excitation of Polar Motion}

[15] Besides the Chandler wobble, the atmosphere also excites wobbles with periods characterized by the various timescales occurring in the atmosphere such as seasonal or diurnal cycle. The equation of polar motion (wobble) predicts the change in the instantaneous pole position $m$ in response to perturbations of inertia products due to mass redistribution and motion. It can be deduced from the linearized Liouville equations, which can be expressed as

$$
\begin{aligned}
\widetilde{A} \Omega \dot{m}_{x}+ & \Omega^{2}(C-\widetilde{B}) m_{y}+\dot{h}_{x}-\Omega h_{y}+\left(1+k_{2}^{\prime}\right) \Omega \dot{c}_{13} \\
& -\left(1+k_{2}^{\prime}\right) \Omega^{2} c_{23}=0
\end{aligned}
$$

$$
\begin{aligned}
\widetilde{B} \Omega \dot{m}_{y}- & \Omega^{2}(C-\widetilde{A}) m_{x}+\dot{h}_{y}+\Omega h_{x}+\left(1+k_{2}^{\prime}\right) \Omega \dot{c}_{23} \\
& +\left(1+k_{2}^{\prime}\right) \Omega^{2} c_{13}=0
\end{aligned}
$$

by extending the method of Barnes et al. [1983] for a biaxial planet to a triaxial body (see Van Hoolst [2007] for the derivation for a triaxial body). The difference of equations (10) and (11) with those for a biaxial body arises solely from the inequality of $\widetilde{A}$ and $\widetilde{B}$. Here, $k_{2}^{\prime}$ is the degree 2 load Love number (see section 3.1). The atmospheric excitation manifests itself in the relative AAM (wind term of the AAM), $h_{x}$ and $h_{y}$, and the incremental inertia elements (pressure term of the AAM divided by $\Omega$ ), $c_{13}$ and $c_{23}$, which are both explained in section 2.3.

[16] The solutions of equations (10) and (11) yield the instantaneous position of the pole as a function of time, which are given by integrating the polar motion at a given frequency over the entire frequency spectrum

$$
m(t)=\sum_{\sigma} m(\sigma) \exp (i \sigma t)
$$

If Titan is entirely solid, the $\mathrm{x}$ and $\mathrm{y}$ component of $m(\sigma)$ are given by

$$
\begin{aligned}
m_{x}(\sigma)= & \frac{\left[(C-\widetilde{B}) \Omega^{2}+\sigma^{2} \widetilde{B}\right]\left[h_{x}+\Omega\left(1+k_{2}^{\prime}\right) c_{13}\right]}{\widetilde{A} \widetilde{B} \Omega\left(\sigma_{C W}^{2}-\sigma^{2}\right)} \\
& +\frac{i C \sigma \Omega\left[h_{y}+\Omega\left(1+k_{2}^{\prime}\right) c_{23}\right]}{\widetilde{A} \widetilde{B} \Omega\left(\sigma_{C W}^{2}-\sigma^{2}\right)}
\end{aligned}
$$

$$
\begin{aligned}
m_{y}(\sigma)= & \frac{\left[(C-\widetilde{A}) \Omega^{2}+\sigma^{2} \widetilde{A}\right]\left[h_{y}+\Omega\left(1+k_{2}^{\prime}\right) c_{23}\right]}{\widetilde{A} \widetilde{B} \Omega\left(\sigma_{C W}^{2}-\sigma^{2}\right)} \\
& +\frac{i C \sigma \Omega\left[h_{x}+\Omega\left(1+k_{2}^{\prime}\right) c_{13}\right]}{\widetilde{A} \widetilde{B} \Omega\left(\sigma_{C W}^{2}-\sigma^{2}\right)}
\end{aligned}
$$

[17] Here, the terms on the right-hand side including $h_{x}$, $h_{y}, c_{13}$ and $c_{23}$ are complex quantities depending on $\sigma$. This solution shows that polar motion in both $\mathrm{x}$ and $\mathrm{y}$ components is resonant at the Chandler wobble frequency as for the classical polar motion solution for a biaxial planet, to which it reduces when $A$ is set equal to $B$.

[18] If Titan's interior harbors a subsurface ocean underneath the outer crust and no coupling between the outer crust and mantle/core is assumed, only the outer crust undergoes polar motion. Therefore, all the momenta of inertia in equations (13) and (14) have to be replaced by those of the outer crust, except the MOI differences in the first term:

$$
\begin{aligned}
m_{x}(\sigma)= & \frac{\left[(C-\widetilde{B}) \Omega^{2}+\sigma^{2} \widetilde{B}_{c}\right]\left[h_{x}+\Omega\left(1+k_{2}^{\prime}\right) c_{13}\right]}{\widetilde{A}_{c} \widetilde{B}_{c} \Omega\left(\sigma_{C W}^{2}-\sigma^{2}\right)} \\
& +\frac{i C_{c} \sigma \Omega\left[h_{y}+\Omega\left(1+k_{2}^{\prime}\right) c_{23}\right]}{\widetilde{A}_{c} \widetilde{B}_{c} \Omega\left(\sigma_{C W}^{2}-\sigma^{2}\right)}
\end{aligned}
$$

$$
\begin{aligned}
m_{y}(\sigma)= & \frac{\left[(C-\widetilde{A}) \Omega^{2}+\sigma^{2} \widetilde{A}_{c}\right]\left[h_{y}+\Omega\left(1+k_{2}^{\prime}\right) c_{23}\right]}{\widetilde{A}_{c} \widetilde{B}_{c} \Omega\left(\sigma_{C W}^{2}-\sigma^{2}\right)} \\
& +\frac{i C_{c} \sigma \Omega\left[h_{x}+\Omega\left(1+k_{2}^{\prime}\right) c_{13}\right]}{\widetilde{A}_{c} \widetilde{B}_{c} \Omega\left(\sigma_{C W}^{2}-\sigma^{2}\right)}
\end{aligned}
$$

Here, the internal parameters $\left(k_{2}^{\prime}, \sigma_{C W}\right)$ for the ocean case have to be used. The $\underset{\sim}{\mathrm{A} O I}$ of the deformed crust, $\widetilde{A}_{c}$ and $\widetilde{B}_{c}$, are calculated from $\widetilde{A}$ and $\widetilde{B}$, respectively, analogously to equation (8). In equations (15) and (16) the MOI differences $C-\widetilde{A}$ and $C-\widetilde{B}$ are not replaced by the differences of the MOI of the crust because full decoupling implies a spherically symmetric interior.

[19] The variable $m(t)$ is expressed in radians, which can be converted to meters by multiplying by Titan's radius. Equation (12) is solved numerically with a spectral resolution of $\Delta \sigma=1.69 \times 10^{-9} \mathrm{~Hz}$.

[20] In our baseline calculation we assume that there is no interaction between Titan's polar motion and Saturn or between the outer crust and the subsurface ocean and mantle/core. This is not correct since there are additional torques that would counteract the polar motion. Depending on the assumed interior structure the additional torques comprise Saturn's gravitational torque (external coupling) on Titan [Van Hoolst et al., 2009], the gravitational and pressure torque between the outer crust above a subsurface ocean (internal coupling) [Karatekin et al., 2008; Van Hoolst et al., 2009] or the elastic torque acting on the crust [Goldreich and Mitchell, 2010]. We discuss separately in section 4 how these additional torques may affect our model results.

\subsection{Atmospheric Forcing}

[21] In this work only the forcing of Titan's polar motion by the atmosphere is considered, although we do not a priori rule out the presence of other forcing mechanisms on Titan. We apply the angular momentum approach, which means that the atmospheric torque acting on Titan is not explicitly calculated but derived from the time derivative of the equatorial AAM predicted by a GCM. The alternative, torque approach is known to be more delicate than 
the angular momentum approach [de Viron and Dehant, 1999].

[22] We use the time series of the equatorial AAM predicted by the three-dimensional Titan GCM of Tokano [2010]. The time series covers a full Titan year and is sampled 24 times per Titan day in order to resolve the diurnal cycle. This GCM does not contain topography and no geographical variation in the surface properties (albedo, thermal inertia etc.). The only mechanism to cause longitudinal anisotropy is Saturn's gravitational tide. It was shown by Tokano [2010] that the tilt of the AAM vector in the stratosphere predicted by the GCM roughly agrees with Cassini data [Achterberg et al., 2008], while the axial AAM in the stratosphere is underestimated. Since the tilt angle is given by $\tan ^{-1}$ of the ratio of the equatorial to the axial AAM this could mean that the equatorial AAM in the stratosphere is underestimated as well. However, the vast majority of the equatorial AAM is contributed by tropospheric winds, which are not underestimated by this GCM.

[23] The two equatorial components of the relative AAM are given [Tokano, 2010] by

$$
\begin{aligned}
& h_{x}=-\frac{R^{3}}{g} \int_{0}^{p_{s}} \int_{-\pi / 2}^{\pi / 2} \int_{0}^{2 \pi}(u \sin \phi \cos \lambda-v \sin \lambda) \cos \phi d \lambda d \phi d p \\
& h_{y}=-\frac{R^{3}}{g} \int_{0}^{p_{s}} \int_{-\pi / 2}^{\pi / 2} \int_{0}^{2 \pi}(u \sin \phi \sin \lambda+v \cos \lambda) \cos \phi d \lambda d \phi d p
\end{aligned}
$$

The two equatorial components of incremental inertia elements are given by

$$
\begin{aligned}
& c_{13}=-\frac{R^{4}}{g} \int_{-\pi / 2}^{\pi / 2} \int_{0}^{2 \pi} p_{s} \sin \phi \cos ^{2} \phi \cos \lambda d \lambda d \phi \\
& c_{23}=-\frac{R^{4}}{g} \int_{-\pi / 2}^{\pi / 2} \int_{0}^{2 \pi} p_{s} \sin \phi \cos ^{2} \phi \sin \lambda d \lambda d \phi
\end{aligned}
$$

Here, $g=1.354 \mathrm{~m} \mathrm{~s}^{-2}$ is Titan's gravitational acceleration, $p_{s}$ is the surface air pressure, $u$ is the zonal wind, $v$ is the meridional wind, $\phi$ is the latitude, $\lambda$ is the longitude and $p$ is the air pressure.

[24] The time derivative of $h_{x}$ and $h_{y}$ corresponds to the surface friction torque as a result of tangential stress placed upon the surface by the surface wind. In the absence of topography in the GCM we used, there is no mountain torque associated with the atmospheric pressure on the topography. Therefore, we note that the atmospheric torque in our model may be underestimated to some extent.

\section{Results}

\subsection{Parameters of Titan's Interior and Chandler Wobble}

[25] The polar motion of a planet/moon strongly depends on the parameters that describe the planetary interior. In the case of Titan, some of the parameters are reasonably well known, while others are uncertain or even totally unknown.

[26] The axial MOI factor of Titan was determined by gravity measurements of the Cassini radio science [Iess et al., 2010]. An axial MOI factor of 0.34 was derived, so the axial moment of inertia amounts to $C=0.34 M R^{2}=$ $3.03218 \times 10^{35} \mathrm{~kg} \mathrm{~m}^{2}$. With Titan's gravity coefficients $J_{2}$, $S_{22}$ and $C_{22}$ determined by Iess et al. [2010], equations (1), (2), and (9) yield equatorial MOI of $A=3.03172 \times 10^{35} \mathrm{~kg} \mathrm{~m}^{2}, B=$ $3.03208 \times 10^{35} \mathrm{~kg} \mathrm{~m}^{2}$ and polar flattening of $\alpha=9.355 \times 10^{-5}$.

[27] In the case of an internal ocean the unknown thickness of the outer crust controls several model parameters. Among various models of Titan's interior, the model of Grindrod et al. [2008] predicts the largest crustal thickness $(176 \mathrm{~km})$. Medium crustal thicknesses of $90 \mathrm{~km}$ and $100 \mathrm{~km}$ are suggested by Mitri and Showman [2008] and Nimmo and Bills [2010], respectively. Older models preferred a crustal thickness around $70 \mathrm{~km}$ [Grasset et al., 2000; Sohl et al., 2003; Tobie et al., 2005]. The smallest nominal crustal thickness $(10-50 \mathrm{~km})$ was suggested by Béghin et al. [2009] based on measurements of the Schumann resonance by the Huygens probe.

[28] Figure 1a shows how the ratio $C / C_{c}$ depends on the crustal thickness. Should Titan have a decoupling subsurface ocean, the polar motion amplitude would amplify by at least a factor of 5 compared to Titan without such an ocean. As long as the crust has a relatively large thickness this ratio almost linearly increases with decreasing thickness. However, if the crustal thickness approaches the lower limit, $C / C_{c}$ rapidly grows.

[29] The available gravity data of Cassini do not yet allow a reliable estimation of the degree 2 tidal potential Love number $k_{2}$ [Iess et al., 2010], which is required for the Chandler frequency. Therefore, we adopt the theoretically calculated value after Sohl et al. [2003]. If there is a subsurface ocean, $k_{2}$ increases with decreasing crustal thickness from 0.28 (200 km crustal thickness) to $0.4(20 \mathrm{~km}$ crustal thickness). If there is no internal ocean, $k_{2}=0.03$.

[30] We adopt the load Love number (mass load coefficient) $k_{2}^{\prime}$ from the theoretical work of Sohl et al. [1995]. In the absence of a subsurface ocean $k_{2}^{\prime}=-0.03$ and in the presence of an ocean $k_{2}^{\prime}=-0.83$. The latter number should somewhat depend on the crustal thickness, but there are other uncertainties that affect the number as well. Therefore, we refrain from varying $k_{2}^{\prime}$ with the crustal thickness.

[31] In the presence of a subsurface ocean, mass redistribution of the crust is facilitated, so it largely compensates the atmospheric load, which is given by the pressure term. This is expressed by a load Love number close to -1 , so $1+k_{2}^{\prime}$ is close to zero. If instead no ocean is present, the pressure term is barely compensated.

[32] If there is no subsurface ocean, the Chandler frequency is $3.2513 \times 10^{-10} \mathrm{~Hz}$ after equation (5), corresponding to a Chandler period of 612.4 years, which is 20 times longer than one Titan year (29.5 years, i.e., Saturn's orbital period). As long as the AAM variation is characterized by seasonal or subseasonal oscillations, a resonance between the atmospherically excited wobble and the Chandler wobble is unlikely in the no-ocean case.

[33] We can quantify how the triaxiality and deformation of Titan change the free wobble period of Titan. If Titan 
a) MOI of Titan/MOI of the crust

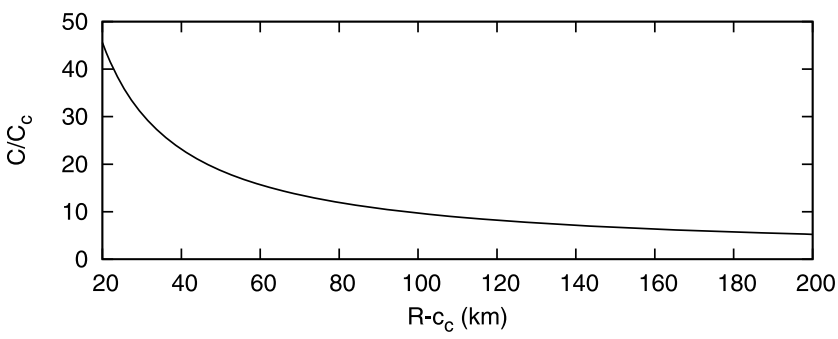

b) Chandler wobble frequency

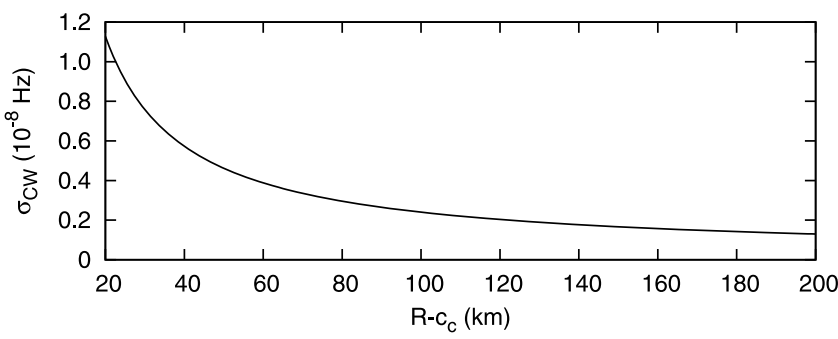

c) Chandler wobble period

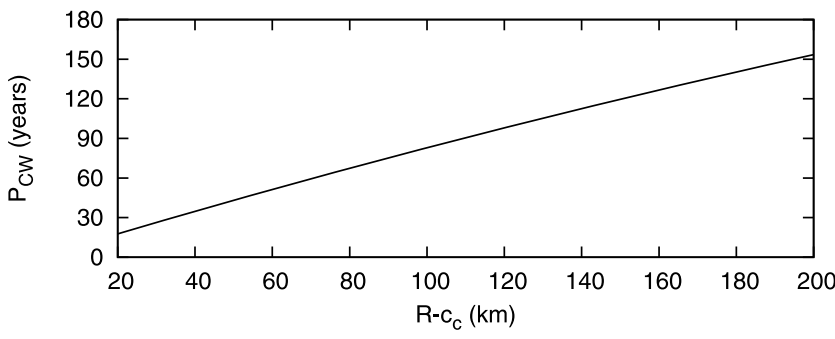

Figure 1. Parameters of Titan's interior relevant for the polar motion as a function of the crustal thickness $\left(R-c_{c}\right)$ above a putative subsurface ocean. (a) Ratio of momentum of inertia of whole Titan to that of the outer crust only. The amplitude of the polar motion amplifies by this factor if Titan has a decoupling internal ocean. $C_{c}$ is obtained from equation (8). (b and c) Chandler wobble frequency and period.

were a biaxial, rigid body, the free wobble period (Euler period) would be

$$
P_{E}=\frac{2 \pi}{((C-\bar{A}) / \bar{A}) \Omega}
$$

where $\bar{A}=(A+B) / 2 . P_{E}$ amounts to 472.8 years.

[34] The free wobble period of a triaxial, rigid body is given by equation (5), where $\widetilde{A}$ and $\widetilde{B}$ are replaced by $A$ and $B$, respectively. This amounts to 599.6 years, i.e., the triaxiality lengthens the free wobble period by 126.8 years. The deformation of Titan lengthens the free wobble period by another 12.8 years. As a whole the deformation in Titan's interior and the triaxial shape lengthen the free wobble period by $\sim 30 \%$.

[35] However, if there is a subsurface ocean, the Chandler wobble of the crust accelerates because the crust has a smaller MOI than entire Titan. Generally, a subsurface ocean reduces the Chandler period by an order of magnitude (Figure 1c). The Chandler period decreases with decreasing crustal thickness. It turns out that there is a certain thickness at which the Chandler period coincides with the annual period of Titan. This coincidence is found at a crustal thickness of $\sim 35 \mathrm{~km}$. In such a case a substantial modification of the polar motion by the Chandler wobble can be expected, as shown below.

\subsection{Atmospheric Forcing}

[36] In our study the temporal variation in the equatorial AAM is the only forcing mechanism of Titan's polar motion. The time series of the relative AAM used in this study were presented in Figures 1 and 3 of Tokano [2010]. The time series of $c_{13}$ and $c_{23}$ times $\Omega$ were presented in Figure 4 of Tokano [2010]. $h_{x}$ and $h_{y}$ undergo a diurnal oscillation due to thermal tides. The amplitude of the diurnal oscillation varies semiannually and becomes maximal twice per Titan year between solstice and subsequent equinox.

[37] Figure 2 shows the atmospheric forcing in the frequency domain used in equations (13) to (16). It elucidates how much the atmosphere forces polar motion at which frequency. The wind term exhibits the largest peak at the diurnal period $\left(4.56 \times 10^{-6} \mathrm{~Hz}\right.$ frequency). Secondary, broad peaks are seen in the low-frequency band. They reflect atmospheric waves present in the model, which are neither thermal nor gravitational tides. The most distinct peaks in the low-frequency band are found at the semiannual $(\sigma=1.35 \times$ $\left.10^{-8} \mathrm{~Hz}\right)$ and annual $\left(6.76 \times 10^{-9} \mathrm{~Hz}\right)$ period. The semiannual variation of the amount of the equatorial AAM of Titan is mainly caused by the reversal of the Hadley circulation twice per Titan year that covers both the troposphere and stratosphere [Tokano, 2010].

[38] The pressure term is 2 orders of magnitude smaller than the wind term, so it contributes much less to the polar motion of Titan. In the presence of a subsurface ocean the pressure contribution to the polar motion is further reduced by the load Love number $k_{2}^{\prime}$ close to -1 . The seasonal forcing (at low frequencies) is as strong as the diurnal forcing and there is another broad peak around 10 Titan days. While negative frequencies are negligible in the wind term, they are present in the pressure term. They are caused by the fact that gravitational atmospheric tides contain both westward and eastward propagating modes. However, because of the overall weakness of the pressure term forcing on Titan a retrograde wobble does not become apparent.

\subsection{Polar Motion}

[39] In this section we present and discuss the polar motion predicted under the hypothetical assumption that besides the atmospheric torque no external and internal torques and dissipation exist. This serves as a baseline model to which further studies on additional torques can be referred. Some discussion on additional torques follows in section 3.4. Therefore, the model prediction in this section should not be directly used for the interpretation of possible observations of Titan's polar motion.

[40] We calculated the polar motion for a period of 1 Titan year for various models of Titan's interior (no subsurface ocean, with subsurface ocean, three different crustal thicknesses). Polar motion with a period of longer than 1 Titan year is neglected since the atmospheric data themselves cover only 1 Titan year, i.e., the lower limit of $\sigma$ in equation (12) is $6.76 \times$ $10^{-9} \mathrm{~Hz}$. The upper limit is $\sigma=1.09 \times 10^{-4} \mathrm{~Hz}$, corresponding 
a) Wind term, $x$

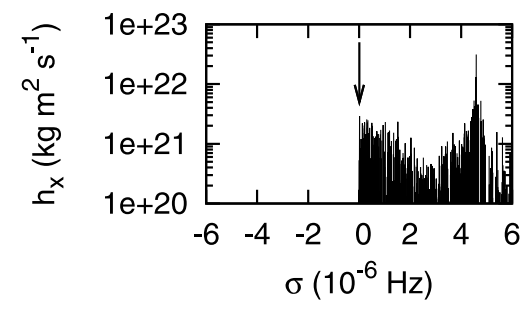

b) Wind term, y

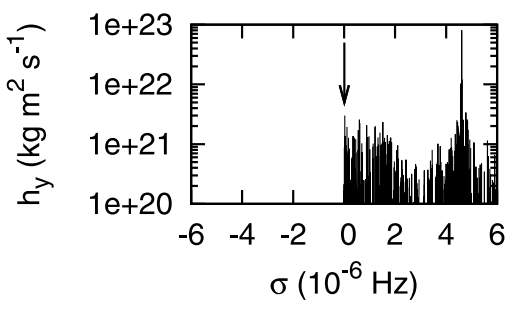

c) Pressure term, $x$

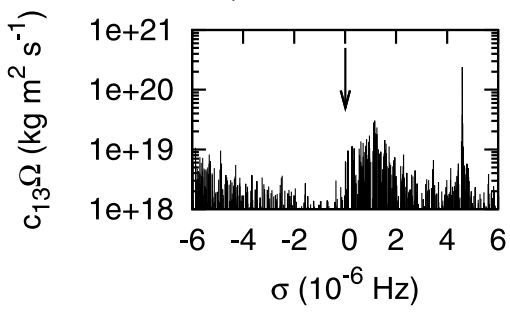

d) Pressure term, y

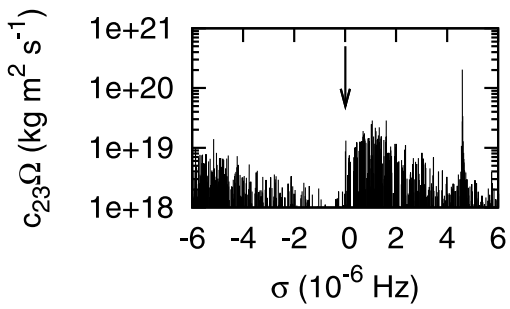

Zoom of a)

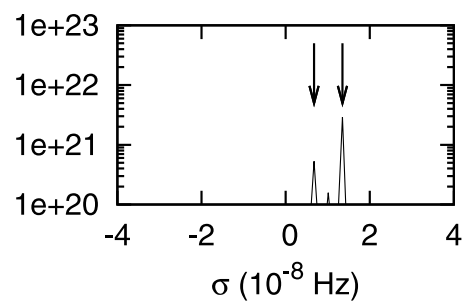

Zoom of b)

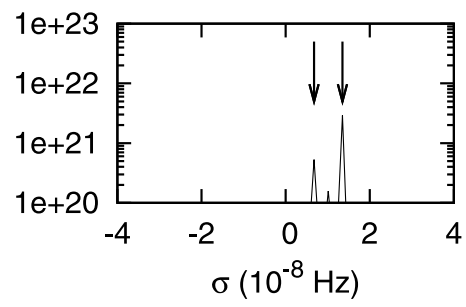

Zoom of c)

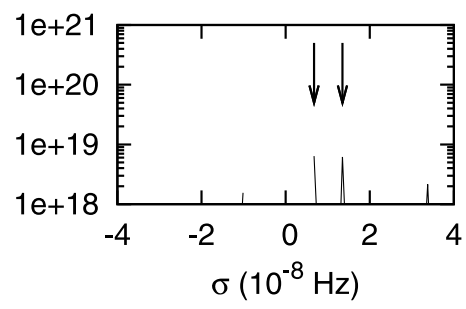

Zoom of d)

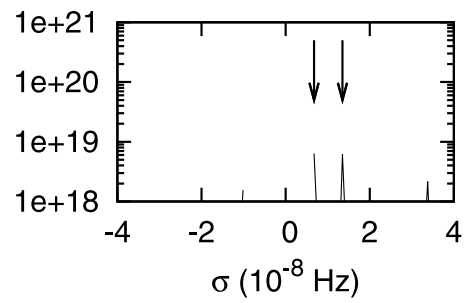

Figure 2. (left) Equatorial components of the AAM in the frequency domain. (a and b) The wind term of the AAM $\left(h_{x}\right.$ and $\left.h_{y}\right)$. (c and d) The pressure term of the AAM $\left(c_{13} \Omega\right.$ and $c_{23} \Omega$ ). The term $\sigma=4.56 \times 10^{-6} \mathrm{~Hz}$ corresponds to the diurnal frequency, $\sigma \sim 1.35 \times 10^{-8} \mathrm{~Hz}$ to the semiannual frequency and $6.76 \times 10^{-9} \mathrm{~Hz}$ to the annual frequency on Titan, latter of which is marked by an arrow. Positive and negative $\sigma$ causes prograde (anticlockwise) and retrograde (clockwise) rotation of the rotation axis around the geographical reference pole. (right) A zoom of the low-frequency band.

to $1 / 24$ Titan day. We do not predict the possible interannual variability in the polar motion since the significance of interannual variability in the atmospheric circulation of Titan is yet unclear.

[41] The solution of the polar motion equation is an initial value problem with a possible nonzero phase with respect to $\sigma t$ in equation (12). In other words, it is not possible to unambiguously determine the absolute coordinates of $\mathrm{x}$ and $\mathrm{y}$ as a function of season unless the initial position is known. In the lack of observational data we started the simulation arbitrarily from $L_{S}=0^{\circ}$ at $t=0$. If the simulation is started from an another season, the starting point would be at another location on the displayed polar motion path.
[42] The calculation shows that the polar motion depends both qualitatively and quantitatively on the interior structure assumed. Figure 3 shows the time series of the polar motion. A superposition of high-frequency and low-frequency oscillations can be readily recognized in each Titan model, although the low-frequency oscillations do not necessarily exhibit a simple sinusoidal shape. In order to better understand the shape of the polar motion and its dependence on the crustal thickness we also decomposed the polar motion time series into different frequency bands, which are shown in Figures 4 and 5 .

[43] Figure 4 elucidates that the high-frequency oscillation is a diurnal wobble caused by thermal tides described 
No ocean, $x$

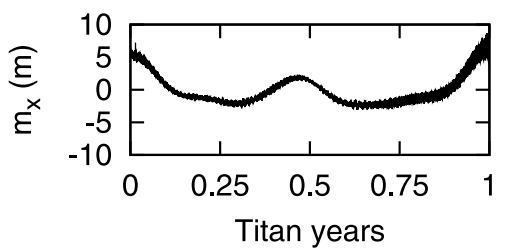

Ocean with $100 \mathrm{~km}$ crust, $x$

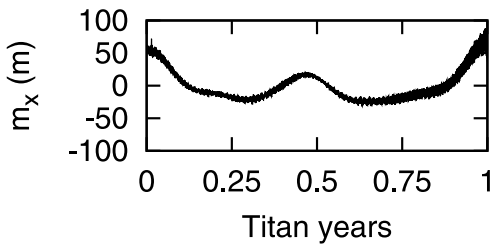

Ocean with $70 \mathrm{~km}$ crust, $\mathrm{x}$

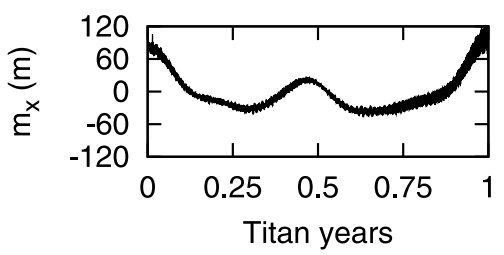

Ocean with $40 \mathrm{~km}$ crust, $\mathrm{x}$

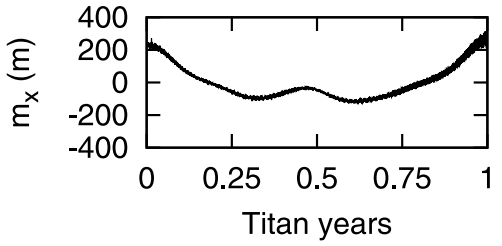

Ocean with $20 \mathrm{~km}$ crust, $\mathrm{x}$

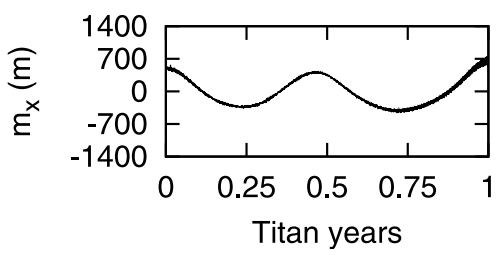

No ocean, y

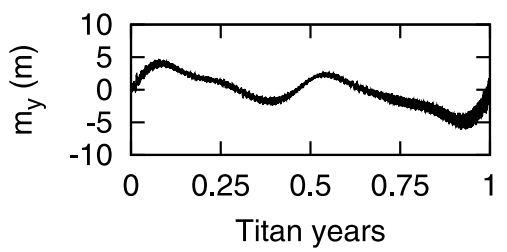

Ocean with $100 \mathrm{~km}$ crust, y

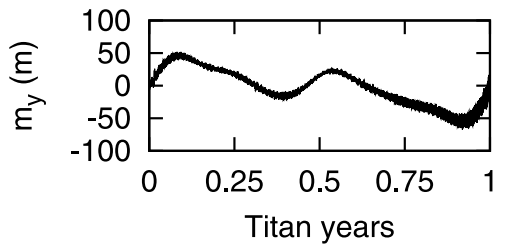

Ocean with $70 \mathrm{~km}$ crust, y

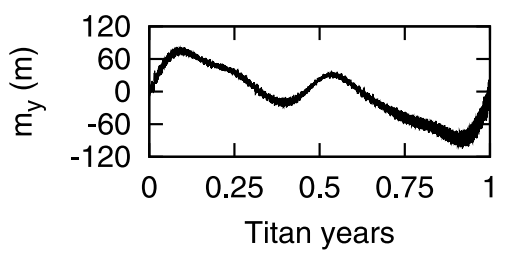

Ocean with $40 \mathrm{~km}$ crust, y

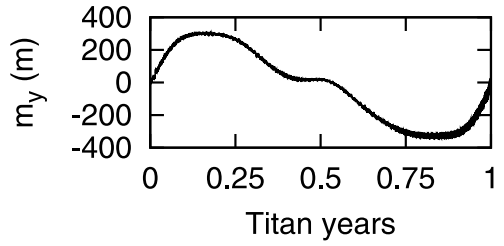

Ocean with $20 \mathrm{~km}$ crust, y

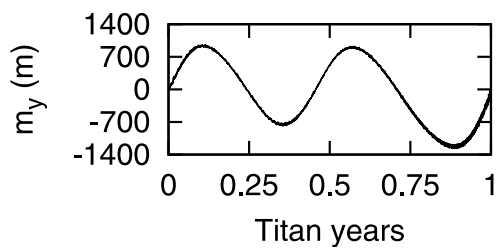

Figure 3. Time series of the polar motion for different interior models. The curves show the instantaneous position of the rotation axis (deviation from Titan's figure axis in meters) as a function of time. The left and right column show the $\mathrm{x}$ and $\mathrm{y}$ component, respectively. The first row shows the result for Titan without a subsurface ocean, the second through fifth rows show the results for Titan with a subsurface ocean of different crustal thickness. The polar motion amplitude has been converted from radians to meters for convenience. The corresponding paths of the polar motion are depicted in Figures 6 to 8 .

by Tokano [2010]. In the absence of a subsurface ocean the amplitude of the diurnal wobble is of the order of a meter. However, the amplitude (polar motion radius) of the diurnal wobble is modulated seasonally as with the tilt angle of the AAM vector [Tokano, 2010]. If Titan has a decoupling subsurface ocean, the amplitude of the diurnal wobble is 1 order of magnitude larger because only the outer crust responds immediately to the atmospheric forcing. The amplitude gradually grows from $\sim 5 \mathrm{~m}$ at a crustal thickness of $100 \mathrm{~km}$ to $\sim 20 \mathrm{~m}$ at a crustal thickness of $30 \mathrm{~km}$. This amplitude increase mostly reflects the increase of $C / C_{c}$ shown in Figure 1a. The tidal potential Love number has little influence since $c_{13}$ and $c_{23}$ are small on Titan. Also the
Chandler wobble has no influence on the diurnal wobble because the periods of both wobbles differ by several orders of magnitude from each other.

[44] The medium-frequency band (Figure 5, left) is dominated by the semiannual wobble (two waves per Titan year) associated with the periodical reversal of the Hadley circulation, which occurs twice per Titan year around equinoxes. The low-frequency band almost exclusively consists of the annual wobble with one wave per Titan year. This analysis shows that the semiannual and annual wobble have comparable amplitudes although the relative importance of each wobble depends on the interior structure as discussed below. In these two frequency bands the wobble has an amplitude 


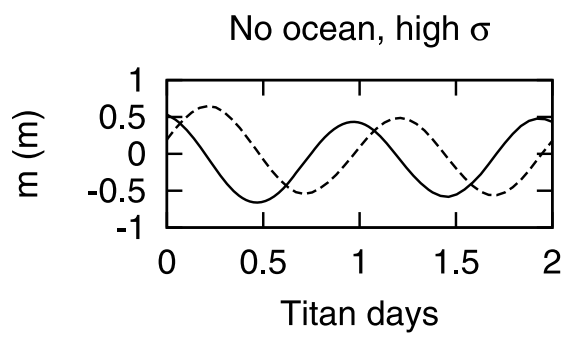

Ocean with $100 \mathrm{~km}$ crust, high $\sigma$

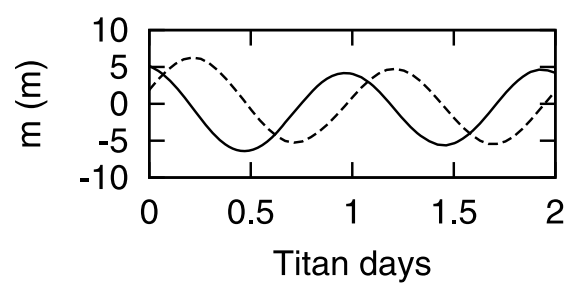

Ocean with $70 \mathrm{~km}$ crust, high $\sigma$

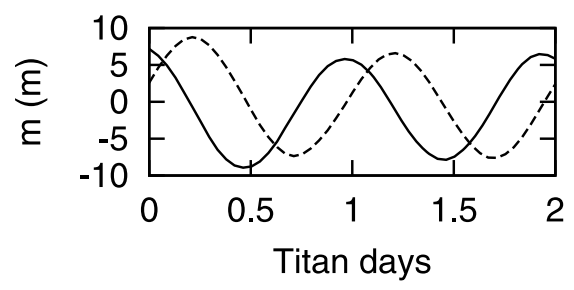

Ocean with $40 \mathrm{~km}$ crust, high $\sigma$

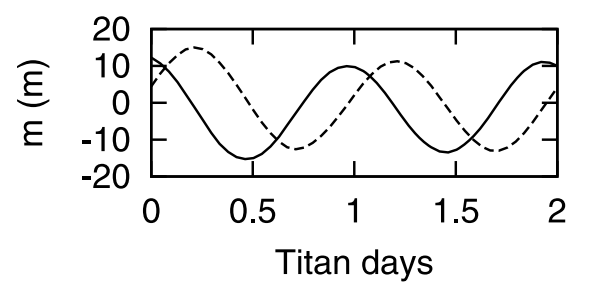

Ocean with $20 \mathrm{~km}$ crust, high $\sigma$

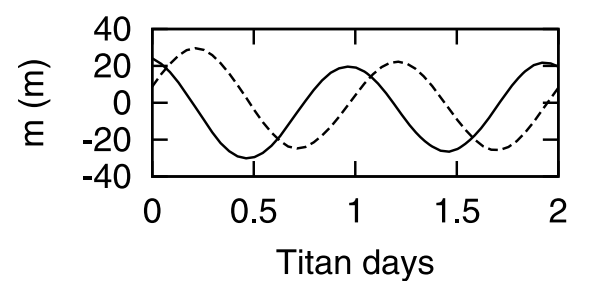

Figure 4. Zoom of Figure 3 at the season $L_{S}=90^{\circ}$ (northern summer solstice) for 2 consecutive Titan days showing the diurnal wobble. The solid and dashed line show the $\mathrm{x}$ and $\mathrm{y}$ component, respectively.

larger than the diurnal wobble although the forcing is weaker. The reason for the larger wobble is the closer proximity of the forcing frequency $\sigma$ to the Chandler frequency $\sigma_{C W}$.

[45] The overall shape in Figure 3 can be basically understood as a superposition of the annual, semiannual and diurnal wobbles. The magnitudes of two consecutive semiannual wobbles slightly differ from each other because of Titan's asymmetric seasons caused by Saturn's orbital eccentricity. Interestingly the shape of the polar motion depends on the crustal thickness. If Titan is entirely solid or if there is a decoupling subsurface ocean below a relatively thick crust (e.g., $100 \mathrm{~km}$ or more), the $\mathrm{x}$ and $\mathrm{y}$ component of the polar motion have similar amplitudes. However, if the crust becomes thinner, the y component increases at the expense of the $\mathrm{x}$ component. This can be already recognized in the $70 \mathrm{~km}$ case, but is much more pronounced if the crust is thinner. The reason for this asymmetry between $\mathrm{x}$ and $\mathrm{y}$ is the fact that $C-\widetilde{A}$ is 4 to 5 times larger than $C-\widetilde{B}$ in equations (15) and (16). The terms containing these MOI differences become relatively more important if the crust becomes thinner, i.e., $C_{c}, \widetilde{A}_{c}$ and $\widetilde{B}_{c}$ are small. At crustal thicknesses larger than $100 \mathrm{~km}$ (not shown) the result is rather similar to that of the $100 \mathrm{~km}$ case, but slowly approaches the solid Titan case. Also, if the outer crust is not fully decoupled from the interior by the ocean, the resulting polar motion would be closer to that of the solid Titan case.

[46] A mathematical singularity in the polar motion equation would occur, should the crustal thickness be about $35 \mathrm{~km}$. In this particular situation $\sigma=\sigma_{C W}$ for the annual forcing period, i.e., a resonance occurs between the annual period and the Chandler period. According to equations (15) and (16) the polar motion amplitude would become infinite. In reality, an infinite polar motion is impossible since dissipation in Titan's interior would damp the polar motion.

[47] The dissipation of the polar motion is characterized by the wobble dissipation factor $Q$, which contains information about the budget and processes of kinetic energy dissipation in the planetary interior. The Chandler wobble amplitude decays to $e^{-1}$ of its original value in a timescale $t_{d}=2 Q / \sigma_{C W}$ [Gross, 2009]. It is common practice to estimate $Q$ from comparisons between the observed and modeled Chandler wobble because the dissipation mechanisms are not well understood. Unfortunately, in the lack of observational data and theoretical studies about the wobble dissipation in an icy crust we are unable to estimate $Q$ at this stage. It is even unclear if $Q$ would be of the same order of magnitude as for the Earth, which varies between 40 and 180 [Gross, 2009].

[48] An influence of a resonance with the Chandler wobble can be seen in the case with a subsurface ocean underneath a thin crust, exemplified here by the $40 \mathrm{~km}$ and $20 \mathrm{~km}$ case. The predicted wobble in the $40 \mathrm{~km}$ case basically consists of one sine curve per Titan year. This is a manifestation of the resonance between the annual atmospheric forcing and the Chandler wobble, whose period is about 30 years (Figure 1c), corresponding to 1 Titan year. The resonance with the Chandler wobble amplifies the annual wobble, while the semiannual wobble is not affected. In contrast to this, the $20 \mathrm{~km}$ case shows a resonance between the semiannual wobble and the Chandler wobble. Therefore, there are two large sine curves per Titan year, while the annual wobble is of minor importance.

[49] Figures 6 to 8 show the calculated paths of the polar motions, for which the time series are shown in Figure 3. In the no-ocean case (Figure 6) the polar motion path consists of anticlockwise rotating small circles, which themselves spiral anticlockwise with a larger radius around the reference pole. Since the amplitudes of the diurnal and semiannual wobble do not strongly differ from each other they are sometimes intermingled. Furthermore, the wobble as a whole is rather circular. 
No ocean, medium $\sigma$

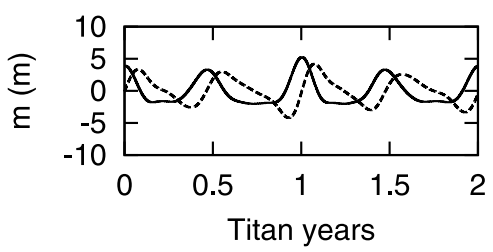

Ocean with $100 \mathrm{~km}$ crust, medium $\sigma$

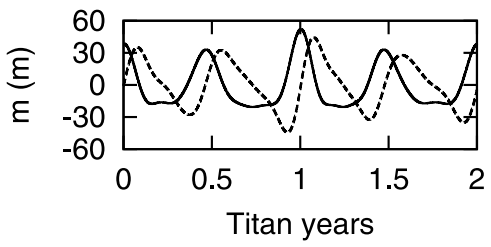

Ocean with $70 \mathrm{~km}$ crust, medium $\sigma$

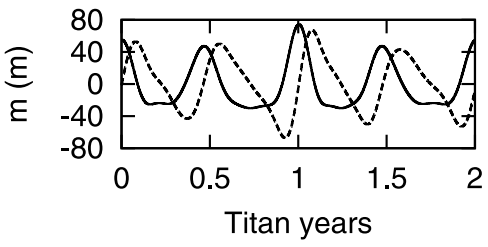

Ocean with $40 \mathrm{~km}$ crust, medium $\sigma$

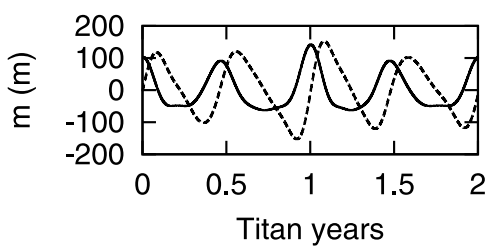

Ocean with $20 \mathrm{~km}$ crust, medium $\sigma$

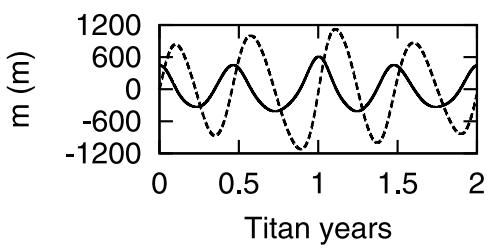

No ocean, low $\sigma$

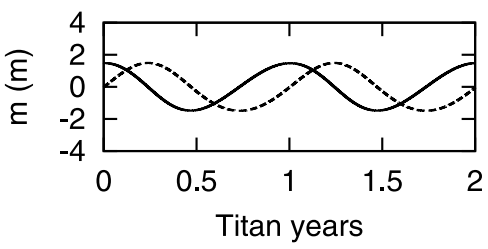

Ocean with $100 \mathrm{~km}$ crust, low $\sigma$

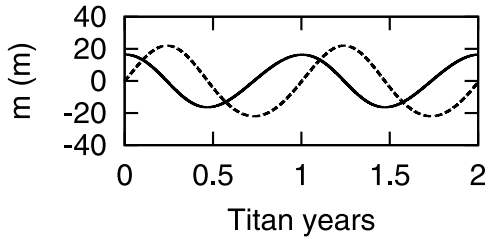

Ocean with $70 \mathrm{~km}$ crust, low $\sigma$

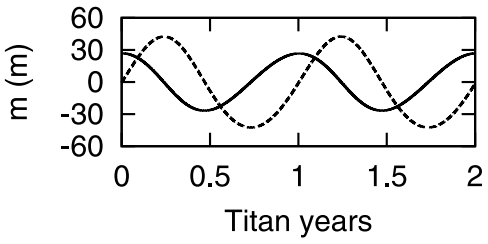

Ocean with $40 \mathrm{~km}$ crust, low $\sigma$

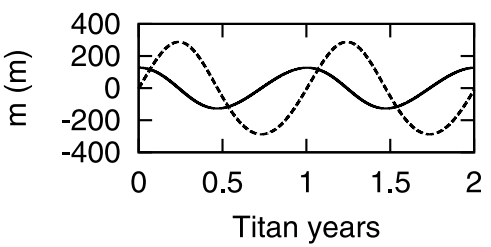

Ocean with $20 \mathrm{~km}$ crust, low $\sigma$

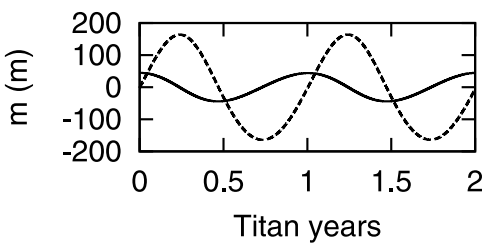

Figure 5. Time series of the polar motion in different frequency bands. (left) The medium-frequency band $\left(10^{-8} \mathrm{~Hz}<\sigma<4.56 \times 10^{-7} \mathrm{~Hz}\right)$ covering forcing periods between 10 Titan days and 456 Titan days (0.676 Titan year). (right) The low-frequency band $\left(\sigma<10^{-8} \mathrm{~Hz}\right)$ covering forcing periods longer than 456 Titan days. The solid and dashed line show the $\mathrm{x}$ and $\mathrm{y}$ component, respectively.

[50] The presence of a subsurface ocean does not substantially change the shape of the polar motion path as long as the crust is $100 \mathrm{~km}$ thick (Figure 7, top) or thicker. However, if the crust is substantially thinner, the polar motion is more elliptical in that it is elongated in the $y$ direction (leading and trailing side or $90^{\circ} \mathrm{W}$ and $90^{\circ} \mathrm{E}$ of Titan). The thinner the crust is the more the elliptical shape pronounced (Figures 7 and 8).

[51] The shape of the polar motion path also depends on the resonance with the Chandler wobble. In the $40 \mathrm{~km}$ case the annual wobble is greatly amplified by the Chandler wobble resonance, so there is basically one large elliptic wobble, which is slightly deformed to a heart shape by the semiannual wobbles, which describe smaller ellipses (Figure 8, top). The $20 \mathrm{~km}$ case, which is characterized by a Chandler wobble resonance with the semiannual wobble, exhibits two large ellipses elongated to the y direction (Figure 8, bottom). The size of these two ellipses differs from each other because of the asymmetric seasons on Titan. In this particular case the amplitude along the $\mathrm{y}$ axis is roughly twice as large as that along the $\mathrm{x}$ axis. The wobble amplitude of the order of $1 \mathrm{~km}$ is $2-3$ orders of magnitude larger than if Titan is entirely solid.

\section{Discussion}

[52] As briefly mentioned at the end of section 2.2, the baseline study does not account for the external and internal gravitational coupling and elastic restoring torque whose influences on Titan's rotation rate variations were investigated by Karatekin et al. [2008], Van Hoolst et al. [2009], and Goldreich and Mitchell [2010]. The external coupling 


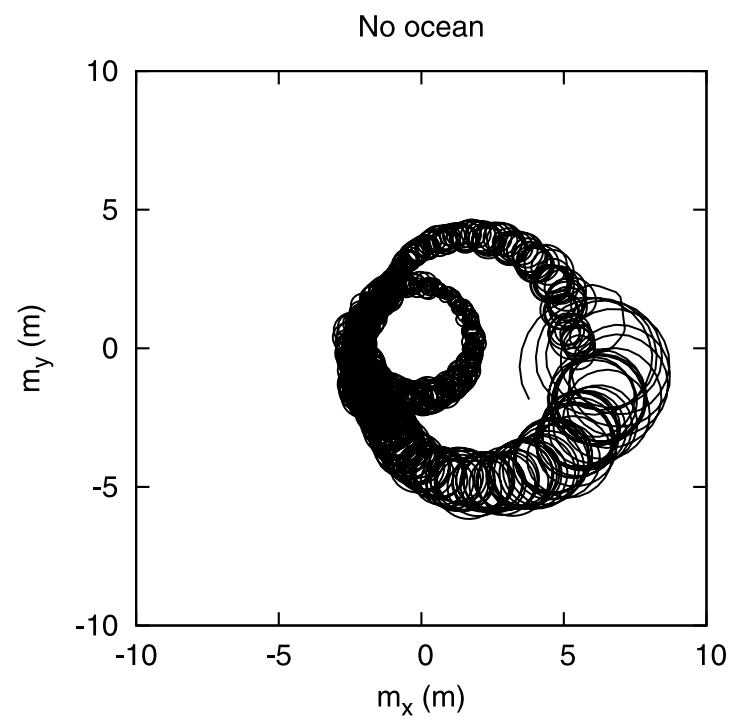

Figure 6. Path of the polar motion (polhody) for the case without a subsurface ocean (solid Titan case) whose time series is shown in Figure 3, no ocean case. We adopt the convention of the International Earth Rotation and Reference Systems Service (IERS), according to which $m_{x}$ is positive toward $0^{\circ}$ longitude (which on Titan is the direction of Saturn) and $m_{y}$ is positive toward $90^{\circ} \mathrm{E}$, so the diagram shows the view from above the pole. The $\mathrm{x}$ and $\mathrm{y}$ range cover $\pm 2.23 \times 10^{-4}$ degrees or $\pm 3.89 \times 10^{-6} \mathrm{rad}$.

(gravitational torque by Saturn) arises from the misalignment of the long axis of Titan with the line joining the mass centers of Titan and Saturn [Van Hoolst et al., 2009]. If Titan has no subsurface ocean, the external gravitational torque by Saturn is the only additional torque that would counteract the polar motion. Its effect on LOD variations of Titan is important and reduces the amplitude of LOD variations by more than an order of magnitude [Van Hoolst et al., 2009]. However, the effect is expected to be much smaller for seasonal polar motion because the averaged torque over one Saturn's orbit is much smaller than the instantaneous torque.

[53] If there is a subsurface ocean, which frictionally decouples the outer crust from the mantle/core, additional torques exist due to Titan's triaxial shape. First, there is a gravitational torque on the crust by the ocean and deeper interior [Karatekinet al., 2008; Van Hoolst et al., 2009]. Secondly, there is a pressure torque caused by variations in the gravitational field due to relative rotation of different internal layers and to orientation change with respect to the direction of Saturn [Van Hoolst et al., 2009]. These two represent the internal coupling between layers. Viscous and electromagnetic interlayer torques are usually neglected on such short timescales as considered in this study.

[54] The internal torques would reduce (in addition to external coupling) the amplitude of the polar motion compared to the results presented in this paper. We here estimate this amplitude reduction based on the effect the couplings have on the LOD variations of Titan induced by the atmosphere [Van Hoolst et al., 2009]. This estimate should be fairly good since similar physical effects are at play, except for the effect of possible resonances with the Chandler wobble. For LOD, we can use equation (40) of Van Hoolst et al. [2009]. At diurnal (high) frequencies, the inertia terms in the right-hand side are several orders of magnitude larger than the coupling terms and the solution reduces to the simple form:

$$
g_{s}=-\frac{\Gamma}{\sigma^{2} C_{c}}
$$

where $\Gamma$ is the atmospheric torque. The effect of internal and external couplings can be neglected at these high frequencies and this is also expected to apply to polar motion.

[55] On the other hand, at seasonal (say, semiannual) frequencies, the internal coupling cannot be neglected and strongly lock the rotations of the crust and the solid interior [see Karatekin et al., 2008; Van Hoolst et al., 2009, Table 2]. This suggests that seasonal polar motion with a subsurface ocean would be of the same order as magnitude as for Titan without an ocean, as is the case for LOD. Generally, the
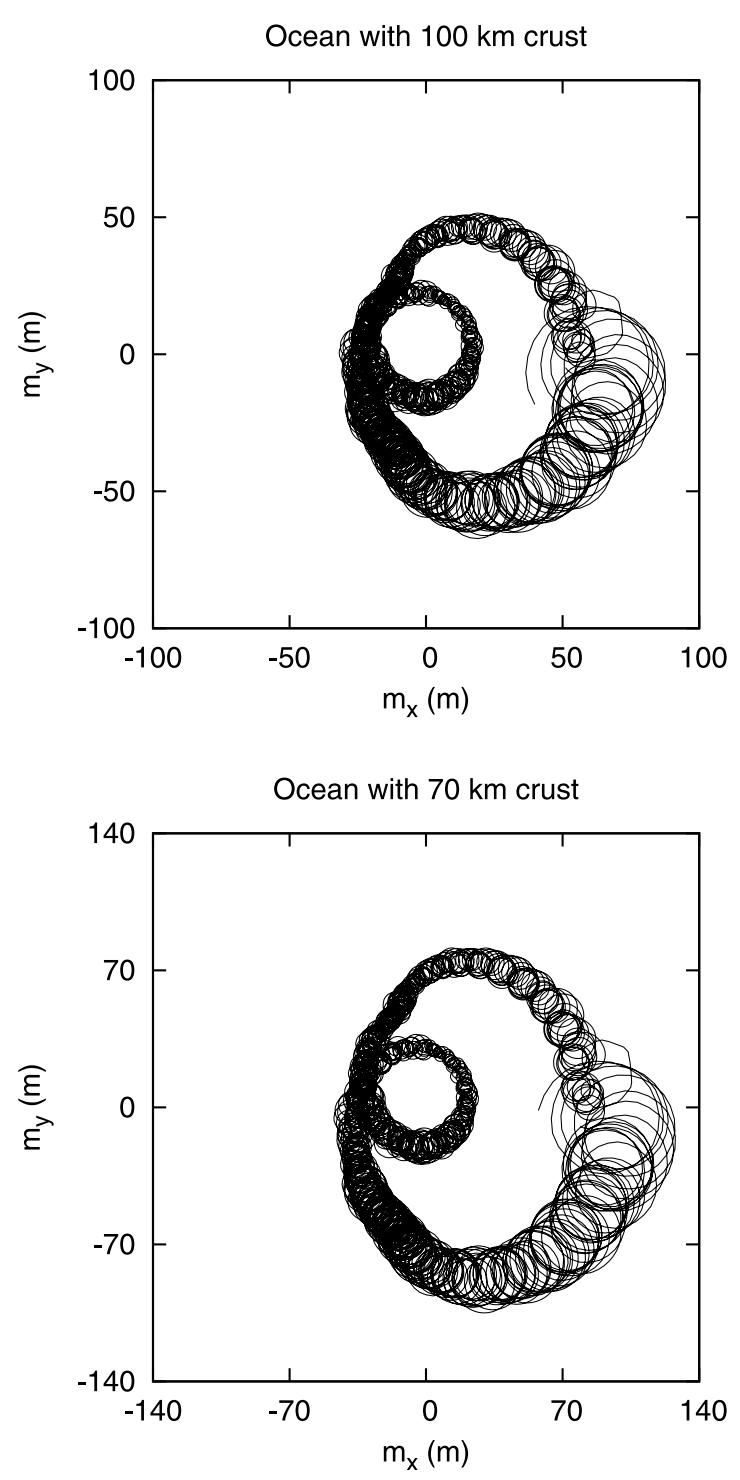

Figure 7. Same as Figure 6 but for the case with a subsurface ocean and a crustal thickness of $100 \mathrm{~km}$ and $70 \mathrm{~km}$. The $\mathrm{x}$ and y range cover (top) $\pm 2.23 \times 10^{-3}$ degrees or $\pm 3.89 \times 10^{-5}$ $\mathrm{rad}$ and (bottom) $\pm 3.12 \times 10^{-3}$ degrees or $\pm 5.45 \times 10^{-5} \mathrm{rad}$. 

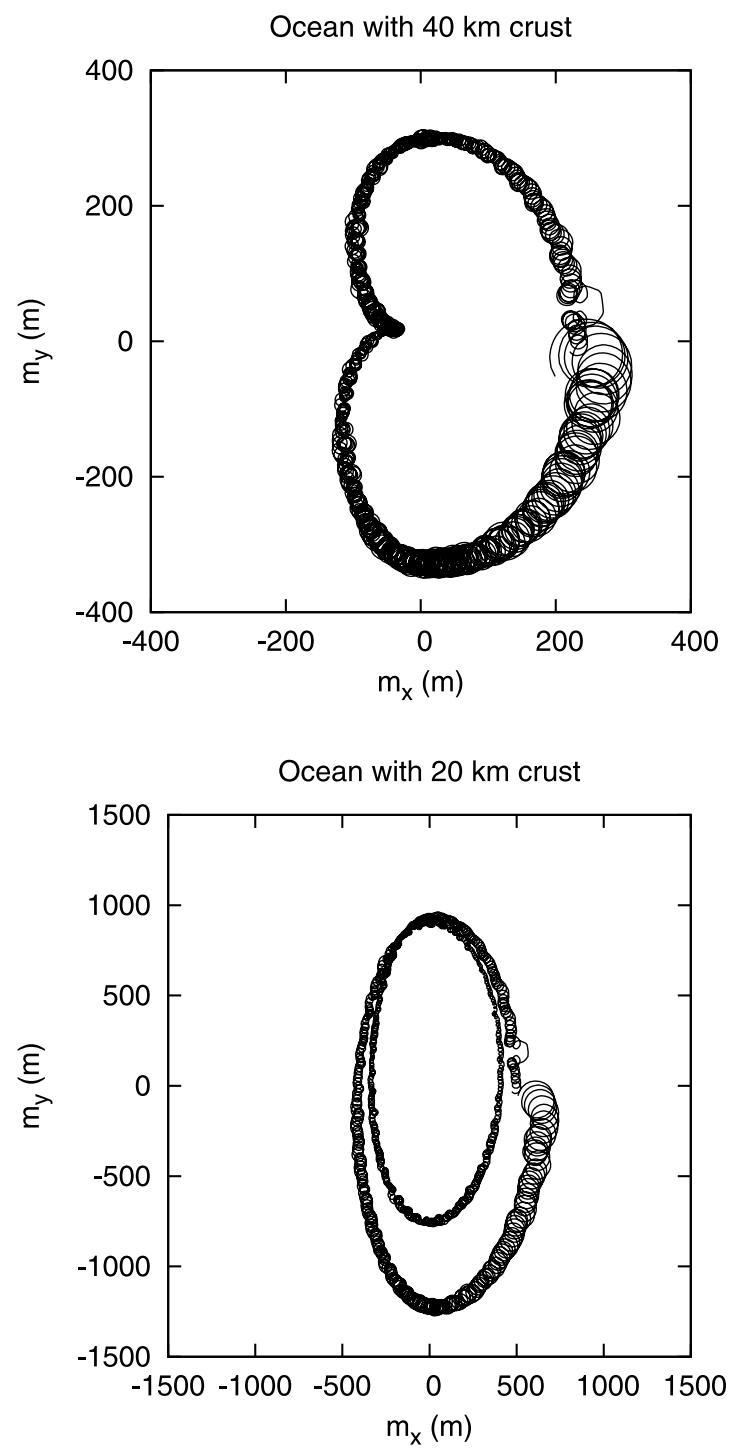

Figure 8. Same as Figure 6 but for the case with a subsurface ocean and a crustal thickness of $40 \mathrm{~km}$ and $20 \mathrm{~km}$. The $x$ and y range cover (top) $\pm 8.9 \times 10^{-3}$ degrees or $\pm 1.55 \times 10^{-4}$ rad and (bottom) \pm 0.033 degrees or $\pm 5.83 \times 10^{-4} \mathrm{rad}$.

additional torques become more important for low frequencies, i.e., the seasonal wobble becomes more strongly attenuated than the diurnal wobble. Hence the diurnal and seasonal wobble are likely to be more intermingled than shown in Figure 8.

[56] The above torques were calculated in the assumption of a rigid crust, neglecting its deformation. Goldreich and Mitchell [2010] introduced an elastic torque related to the idea that incremental rotation with respect to the equilibrium position would lead to deformation of the shell such that it conforms its form to that of the ocean. This reasoning is based on the comparison of elastic energy of deformation to gravitational energy associated with rigid body rotation due to Titan being under the influence of Saturn. Goldreich and Mitchell [2010] obtain a maximum orientation shift in the long axis of Titan in the orbital plane due to the atmosphere of Titan of $7 \times 10^{-4} \mathrm{rad}$ if (only) the elastic torque is taken into account, a subsurface ocean underneath a crust of $100 \mathrm{~km}$ thickness is assumed and the atmospheric torque of Tokano and Neubauer [2005] is used. This is somewhat larger than the maximum orientation shift considering (only) the external and internal coupling $\left(\sim 10^{-4} \mathrm{rad}\right)$ in the rigid crust approximation for the same atmospheric torque [Van Hoolst et al., 2009].

[57] One might think that due to its smaller atmospheric torque the seasonal wobble would be easier to damp than the diurnal wobble. However, the situation for the seasonal wobble is complicated since the orientation of Titan with respect to Saturn changes by $360^{\circ}$ over one Saturn's orbit. It is unlikely that Titan would continuously deform to conform its shape to be symmetric with respect to the direction to Saturn. As a whole, the effect of the elastic torque on the polar motion can be expected to be less than for LOD variations as calculated by Goldreich and Mitchell [2010].

[58] We expect that the inclusion of all these additional torques would not completely erase the polar motion and the polar motion amplitude in the presence of a subsurface ocean is at least as large as that for solid Titan. Possible resonances with the Chandler wobble would still cause a large polar motion despite the additional torques discussed above. It is rather unlikely that the additional torques can substantially modify the Chandler wobble period, as this depends mainly on the effective crust moment of inertia difference. As a supporting argument, Dehant et al. [2003] found that the Chandler wobble period of Mars is rather insensitive to the unknown radius of the inner core underneath a liquid outer core.

[59] Without a substantial modification of the Chandler wobble, a resonance between the seasonal wobble and Chandler wobble would occur despite the additional torques that tend to suppress the overall magnitude of the polar motion. Most importantly, the elliptical shape predicted in the case of thin crusts (Figure 8) would still occur. For this reason the main difference between the solid and ocean case is the shape rather than the amplitude of the seasonal wobble.

[60] In the above consideration of the additional torques, all interior layers of Titan are assumed to have the same triaxial shape. However, the shape of the internal layers (ocean, solid interior) is unknown from observations in contrast to the shape of the surface. If the crust is conductive, the polar crust would be thinner than the equatorial crust because of isostatic compensation [Nimmo and Bills, 2010]. In this configuration the polar subsurface ocean is thicker in comparison with the equatorial ocean, contrary to an ocean with a triaxial shape. The ocean-crust interface would be flat (triaxial global shape) if the lower part of the crust is assumed to be convective [Nimmo and Bills, 2010], but in this case the surface shape (with a slight equatorial depression) would be inconsistent with the observed shape of Titan's surface. A mismatch between the shape of Titan's surface and of the ocean-crust interface would modify the internal coupling to an extent we cannot estimate without knowing the shape/topography of the internal layers. We conclude that there are several unknowns about Titan's interior, which hamper an exact calculation of Titan's polar motion.

[61] We also remark that the atmospheric forcing based on GCM predictions itself is uncertain and additional forcing mechanisms (hydrology, hydrocarbon seas, subsurface ocean) 
are not taken into account in this study. Therefore, one should keep in mind that there are not only effects that would damp Titan's wobble but possibly also additional external forcing mechanisms, which enhance the polar motion.

[62] The Cassini spacecraft is unlikely to detect the diurnal wobble because of its small size and too infrequent Titan flybys for this purpose. However, the large and slow semiannual or annual wobble might be detectable if it is strongly amplified to several kilometers by a resonance with the Chandler wobble. In such an event Titan's outer crust would be thin. More promising would be a monitoring of the polar motion by a long-term Titan orbiter or a polar lander.

\section{Conclusions}

[63] Titan's atmosphere has a large equatorial AAM, which is evidenced by the observed tilt of the symmetry axis of the global circulation from the pole axis [Achterberg et al., 2008] and predicted by a three-dimensional GCM [Tokano, 2010]. This orientation and magnitude of the equatorial AAM are predicted to vary with solar local time and with season. This is accompanied with an exchange of angular momentum with the underlying surface along the equatorial principal axes. Consequently, Titan is forced to perform polar motion (wobble).

[64] This study is a first attempt to quantify Titan's atmospherically excited polar motion using the output of a Titan GCM. We paid attention to the pronounced triaxial shape of Titan in the derivation of the equations of polar motion. Since Titan's interior structure is less known than the interior structure of the terrestrial planets or even many other large moons we calculated the polar motion assuming different interior models. However, parameters of Titan's interior constrained by recent gravity measurements of the Cassini radio science are taken into account.

[65] Polar motion is excited by the transfer of the equatorial component of the AAM with the surface. The atmospheric forcing mainly occurs with diurnal, semiannual and annual periods. The shape and size of Titan's polar motion are shown to depend on the interior structure. If Titan has no subsurface ocean, the diurnal wobble has a typical radius of $1 \mathrm{~m}$, although it is seasonally modulated. Additionally, there are two cycles of semiannual wobbles per Titan year with a radius of about $5 \mathrm{~m}$. Since the amplitudes of the diurnal and seasonal wobbles do not strongly differ from each other the path of the polar motion is intermingled. Although Saturn's gravitational torque counteract the polar motion, the damping of the polar motion may not be as strong as the damping of the LOD variation.

[66] The presence of a subsurface ocean amplifies both the diurnal and seasonal wobble by an order of magnitude and more. The polar motion radius increases with decreasing crustal thickness since the moment of inertia of that portion of Titan that responds to atmospheric forcing becomes smaller. If the crustal thickness is $100 \mathrm{~km}$ and more, which is on the larger side among the theoretical models of Titan's interior, the diurnal wobble has a typical radius of $10 \mathrm{~m}$ and a semiannual and annual wobbles with radii of $50 \mathrm{~m}$.

[67] A resonance between the annual wobble and Chandler wobble would occur if the crustal thickness is close to $35 \mathrm{~km}$. In this particular situation the annual wobble is further amplified, while the superposed semiannual and diurnal wobbles do not change. If the crust is only $20 \mathrm{~km}$ thick, a resonance between the Chandler wobble and semiannual wobble would occur, so two large semiannual wobbles would dominate Titan's wobble. Titan's triaxial shape with a tidal bulge at the sub-Saturnian and anti-Saturnian point causes the polar motion to be elliptical if there is a subsurface ocean and the outer crust is relatively thin. The wobble is elongated along the y axis, i.e., toward the leading point $\left(90^{\circ} \mathrm{W}\right)$ and trailing point $\left(90^{\circ} \mathrm{E}\right)$

[68] If a subsurface ocean is present, the nature of internal coupling between the crust, ocean and solid interior would depend on the assumed mechanical behavior of the crust. If the crust is assumed to be rigid, gravitational and pressure torque act on the crust, while the shell experiences an elastic restoring torque if it is assumed to be deformable. In either case should the torques damp the seasonal wobble more strongly than the diurnal wobble. However, the damping of the polar motion is expected to be generally smaller than the damping of the LOD variation. It is unlikely that the additional torques entirely eliminate the polar motion and polar motion amplitudes of at least the order of meters may be present under any configuration. An elliptical shape rather than the amplitude of the polar motion is probably the best signature of the possible presence of a subsurface ocean in Titan.

[69] Acknowledgments. The work of T.T. was funded by the DFG.

\section{References}

Achterberg, R. K., B. J. Conrath, P. J. Gierasch, F. M. Flasar, and C. A Nixon (2008), Observation of a tilt of Titan's middle-atmospheric superrotation, Icarus, 197, 549-555.

Barnes, R. T. H., R. Hide, A. A. White, and C. A. Wilson (1983), Atmospheric angular momentum fluctuations, length-of-day changes and polar motion, Proc. R. Soc. London, Ser. A, 387, 31-73.

Béghin, C., et al. (2009), New insights on Titan's plasma-driven Schumann resonance inferred from Huygens and Cassini data, Planet. Space Sci., 57, 1872-1888.

Bills, B., and F. Nimmo (2008), Forced obliquity and moments of inertia of Titan, Icarus, 196, 293-297.

Dehant, V., T. Van Hoolst, O. de Viron, M. Greff-Lefftz, H. Legros, and P. Defraigne (2003), Can a solid inner core of Mars be detected from observations of polar motion and nutation of Mars?, J. Geophys. Res., 108(E12), 5127, doi:10.1029/2003JE002140.

de Viron, O., and V. Dehant (1999), Earth's rotation and high frequency equatorial angular momentum budget of the atmosphere, Surv. Geophys., $20,441-462$.

Friedson, A. J., R. A. West, E. H. Wilson, F. Oyafuso, and G. S. Orton (2009), A global climate model of Titan's atmosphere and surface, Planet. Space Sci., 57, 1931-1949.

Goldreich, P. M., and J. L. Mitchell (2010), Elastic ice shells of synchronous moons: Implications for cracks on Europa and non-synchronous rotation of Titan, Icarus, 209, 631-638.

Grasset, O., C. Sotin, and F. Deschamps (2000), On the internal structure and dynamics of Titan, Planet. Space Sci., 48, 617-636.

Grindrod, P. M., A. D. Fortes, F. Nimmo, D. L. Feltham, J. P. Brodholt, and L. Vočadlo (2008), The long-term stability of a possible aqueous ammonium sulfate ocean inside Titan, Icarus, 197, 137-151.

Gross, R. S. (2009), Earth rotation variations - long period, in Treatise on Geophysics, vol. 3, pp. 239-294, edited by T. Herring, Elsevier, Amsterdam.

Iess, L., N. Rappaport, R. A. Jacobson, P. Racioppa, D. J. Stevenson, P. Tortora, J. W. Armstrong, and S. W. Asmar (2010), Gravity field, shape, and moment of inertia of Titan, Science, 327, 1367-1369.

Karatekin, Ö., T. Van Hoolst, and T. Tokano (2008), Effect of internal gravitational coupling on Titan's non-synchronous rotation, Geophys. Res. Lett., 35, L16202, doi:10.1029/2008GL034744.

Konopliv, A. S., C. F. Yoder, E. M. Standish, D.-N. Yuan, and W. L. Sjogren (2006), A global solution for the Mars static and seasonal gravity, Mars orientation, Phobos and Deimos masses, and Mars ephemeris, Icarus, $182,23-50$ 
Lorenz, R. D., B. W. Stiles, R. L. Kirk, M. D. Allison, P. Persi del Marmo, L. Iess, J. I. Lunine, S. J. Ostro, and S. Hensley (2008), Titan's rotation reveals an internal ocean and changing zonal winds, Science, 319 , 1649-1651.

Mitchell, J. L. (2009), Coupling convectively driven atmospheric circulation to surface rotation: evidence for active methane weather in the observed spin rate drift of Titan, Astrophys. J., 692, 168-173.

Mitri, G., and A. P. Showman (2008), Thermal convection in ice-I shells of Titan and Enceladus, Icarus, 193, 387-396.

Munk, W. H., and G. J. F. MacDonald (1960), The Rotation of the Earth, Cambridge Univ. Press, New York.

Nimmo, F., and B. G. Bills (2010), Shell thickness variations and the longwavelength topography of Titan, Icarus, 208, 896-904.

Noyelles, B. (2008), Titan's rotational state. The effects of a forced "free" resonant wobble, Celest. Mech. Dyn. Astron., 101, 13-30.

Noyelles, B., A. Lemaître, and A. Vienne (2008), Titan's rotation. A 3-dimensional theory, Astron. Astrophys., 478, 959-970.

Roman, M. T., R. A. West, D. J. Banfield, P. J. Gierasch, R. K. Achterberg, C. A. Nixon, and P. C. Thomas (2009), Determining a tilt in Titan's northsouth albedo asymmetry from Cassini images, Icarus, 203, 242-249.

Sanchez, B., R. Haberle, and J. Schaeffer (2004), Atmospheric rotational effects on Mars based on the NASA Ames general circulation model: angular momentum approach, J. Geophys. Res., 109, E08005, doi:10.1029/2004JE002254.

Sohl, F., W. D. Sears, and R. D. Lorenz (1995), Tidal dissipation on Titan, Icarus, 115, 278-294.

Sohl, F., H. Hussmann, B. Schwentker, T. Spohn, and R. D. Lorenz (2003), Interior structure models and tidal Love numbers of Titan, J. Geophys. Res., 108(E12), 5130, doi:10.1029/2003JE002044.

Sotin, C., G. Mitri, N. Rappaport, G. Schubert, and D. Stevenson (2009), Titan's interior structure, in Titan From Cassini-Huygens, edited by R. H. Brown et al., pp. 61-73, Springer, New York.
Teanby, N. A., P. G. J. Irwin, and R. de Kok (2010), Compositional evidence for Titan's stratospheric tilt, Planet. Space Sci., 58, 792-800.

Tobie, G., O. Grasset, J. I. Lunine, A. Mocquet, and C. Sotin (2005), Titan's internal structure inferred from a coupled thermal-orbital model, Icarus, 2005, 175, 496-502.

Tokano, T. (2010), Westward rotation of the atmospheric angular momentum vector of Titan by thermal tides, Planet. Space Sci., 58, 814-829.

Tokano, T., and F. M. Neubauer (2005), Wind-induced seasonal angular momentum exchange at Titan's surface and its influence on Titan's length-of-day, Geophys. Res. Lett., 32, L24203, doi:10.1029/ 2005GL024456.

Van den Acker, E., T. Van Hoolst, O. de Viron, P. Defraigne, F. Forget, F. Hourdin, and V. Dehant (2002), Influence of the seasonal winds and the $\mathrm{CO}_{2}$ mass exchange between the atmosphere and polar caps on Mars' rotation, J. Geophys. Res., 107(E7), 5055, doi:10.1029/ 2000JE001539.

Van Hoolst, T. (2007), The rotation of the terrestrial planets, in Treatise on Geophysics, vol. 10, pp. 123-164, edited by T. Spohn, Elsevier, Amsterdam.

Van Hoolst, T., and V. Dehant (2002), Influence of triaxiality and secondorder terms in flattenings on the rotation of terrestrial planets, Phys. Earth Planet. Inter., 134, 17-33.

Van Hoolst, T., N. Rambaux, Ö. Karatekin, and R.-M. Baland (2009), The effect of gravitational and pressure torques on Titan's length-of-day variations, Icarus, 200, 256-264.

T. Tokano, Institut für Geophysik und Meteorologie, Universität zu Köln, Albertus-Magnus-Platz, D-50923 Köln, Germany. (tokano@geo. uni-koeln.de)

T. Van Hoolst and Ö. Karatekin, Royal Observatory of Belgium, Ringlaan 3 Av. Circulaire, B-1180 Brussels, Belgium. 